\title{
Clinico-biochemical, ultrasonographic and pathological findings of hepatic abscess in feedlot cattle and buffaloes
}

\author{
A. M. Abdelaal, Shaimaa M. Gouda and M. Tharwat
}

Department of Animal Medicine, Faculty of Veterinary Medicine, Zagazig University, Zagazig - 44511, Egypt.

Corresponding author: A. M. Abdelaal email: ahmed.abdelaal@zu.edu.eg, abdelaal79@yahoo.com

Received: 16-03-2014, Revised: 05-04-2014, Accepted: 10-04-2014, Published online: 12-05-2014

doi: 10.14202 / vetworld.2014.306-310

How to cite this article: Abdelaal AM, Gouda SM and Tharwat M (2014) Clinico-biochemical, ultrasonographic and pathological findings of hepatic abscess in feedlot cattle and buffaloes, Veterinary World 7(5): 306-310.

\begin{abstract}
Background and aim: Liver abscesses in feedlot cattle have a major economic impact in the beef industry because of liver condemnation and reduced animal performance and carcass yield. The aim of this study was to document the clinicopathological and ultrasonographic findings in cattle and buffaloes with hepatic abscess.

Materials and Methods: Three male feedlot cattle and 5 buffaloes suffered from liver abscesses were investigated. Clinical, hemato-biochemical and Ultrasonographic were performed. Liver abscesses were confirmed through centesis and aspiration under ultrasonographic guidance which yield purulent material. Necropsy and histopathological studies were applied on 3 of them, where ultrasonographic diagnosis was confirmed.

Results: Altered appetite, loss of body weight, dull demeanor and decreased ruminal motility were recorded in all eight animals. Systemic disturbance and feces abnormalities were appeared with variable degrees. Icterus and abdominal pain were observed only in one cattle. Leucocytosis, hypoalbuminaemia and hyperglobulinaemia were reported in all animals while liver enzymes were mildly elevated only in 3 cattle and one buffalo. Abscess appeared by ultrasonography as hypoechoic to echogenic circumscribed mass, pinpoint to $10 \mathrm{~cm}$ in diameter, with or without echogenic wall, located in the right lobe $7^{\text {th }}$ to $12^{\text {th }}$ intercostal spaces. Abscesses were located intra-hepatic in 7 (3 cattle and 4 buffaloes) animals and peri-hepatic (between liver and reticulum) in one buffalo.
\end{abstract}

Conclusions: Ultrasonographic results were of considerable value for ante-mortem diagnosis of hepatic abscesses.

Keywords: abscesses, buffaloes, feedlot cattle, histopathology, liver, ultrasonography.

\section{I ntroduction}

Liver abscesses have a major economic impact on the feedlot industry because of liver condemnation and reduced animal performance and carcass yield [1]. Liver abscesses can occur at all ages and in all types of cattle, including dairy cows, but they have the greatest economic importance for grain-fed cattle [2]. Liver abscesses in feedlot cattle result from aggressive grainfeeding programs and are influenced by a number of dietary and management factors [3]. Generally, the incidence and severity of abscesses increase as roughage level in the diet decreases. High roughage levels promote more stable ruminal fermentation and decrease the variation in feed intake, thereby lowering the incidence of acidosis and rumenitis. The ruminal wall that is damaged from acidity or penetration of foreign objects becomes susceptible to invasion and colonization by $F$. necrophorum and subsequently shed bacterial emboli to the portal circulation, leading to infection and abscess formation [3,4]. Moreover, abscess could be developed as a sequelae of traumatic reticuloperitonitis cattle and buffaloes [5]. Cattle that carry hundreds of small abscesses or several large abscesses, seldom exhibit any clinical signs. Thus, liver abscesses

Copyright: The authors. This article is an open access article licensed under the terms of the Creative Commons Attribution License (http://creativecommons.org/licenses/by/2.0) which permits unrestricted use, distribution and reproduction in any medium, provided the work is properly cited. are detected only at the time of slaughter. Occasionally, cattle may exhibit abdominal pain, or the rupture of a superficial abscess or erosion and perforation of the caudal vena cava could lead to extensive spread and massive infection of other organs and death [6].

Generally, hematology and liver function tests are not reliable indicators of liver abscesses [7]. Ultrasonography has markedly enhanced the diagnosis of hepatic disease in cattle and various hepatic diseases as hepatic abscess, hepatic lipidosis, fascioliasis and caudal vena cava (CVC) thrombosis can be diagnosed through ultrasonography and ultrasound-guided liver biopsy [8-11]. Ultrasonography has been evaluated in diagnosis of liver diseases in human [12,13], in dogs [14], in sheep [15] and equine [16].

Ultrasonography is a useful technique for monitoring the onset and progression of experimentally induced abscesses when the site of injection is known. However, its application in feedlot cattle with naturally developed abscesses is limited. Therefore, this research was planned to document Ultrasonographic Findings of naturally developed Hepatic Abscess feedlot in cattle and buffaloes in relation to clinical, biochemical, and Pathological Findings.

\section{Materials and Methods}

Ethical approval: All study procedures were approved by and in accordance with the rules of animal use and 
care ethical committee of Faculty of Veterinary Medicine, Zagazig University.

Study area and animals: A total of eight male feedlot cattle and buffaloes were examined at the Veterinary Teaching Hospital, Zagazig University, Egypt at March, 2011 to December, 2013. They included 5 buffalo's bull and 3 cattle bull. Animals were aged 1 to 2 years and weighed 300 to $450 \mathrm{~kg}$. Animals were admitted because of altered appetite, weight loss and dull demeanour. Cases had been ill for 10 days to 2 months before admission. All animals underwent a thorough clinical examination as described previously [17], which included general behavior and condition, auscultation of the heart, lungs, rumen and intestine, measurement of heart rate, respiratory rate and rectal temperature, swinging auscultation, percussion auscultation of both sides of the abdomen and rectal examination. Due to the poor prognosis, 3 animals were slaughtered and were thoroughly examined postmortem.

Hematological and biochemical analyses: Two blood samples were collected by puncture of the jugular vein, one on EDTA and the other without an anticoagulant. A complete blood count (hematocrit, red blood cells, total and differential leucocytes) was investigated in a whole blood. After centrifugation of the second blood sample, serum samples were collected and then frozen for later analysis of clinical chemistries. Commercial kits were used to determine the serum concentrations of total protein, albumin, urea nitrogen (BUN) and creatinine. The serum activities of aspartate aminotransferase (AST), Alanine amino transferase (ALT), $\gamma$-glutamyl transferase (GGT), alkaline phosphatase (ALP) and Creatinine kinase (CK) were measured in serum samples.

Ultrasonographic examination and aspiration technique: Ultrasonographic examination was carried out while the animals were standing using 3.5 and 5.0 $\mathrm{MHz}$ convex as well as $6 \mathrm{MHz}$ linear transducers. In preparation for ultrasonography, the intercostal spaces (third to twelve) and the entire abdomen were clipped, shaved and swabbed with alcohol to remove excess oil, and coupling gel was finally applied. Abdominal ultrasonography was carried out as described previously [18]. The peritoneum, rumen, reticulum, omasum, abomasum, spleen, small and large intestines, liver, pancreas and right kidney were examined.

Liver abscesses were confirmed through aspiration of the mass as described previously [18]. Briefly the procedure as follows: under ultrasound guidance, aspiration of hepatic abscesses was made after sterilization by a standard surgical disinfection technique. A $14 \mathrm{G} \mathrm{x}$ 170-mm spinal needle (Kurita Co., Ltd, Tokyo, Japan) was advanced through the skin and guided ultrasonographically towards the lesion and, with a slight thrust, was pushed through. The end of the needle within the abscess was usually visible in the ultrasonographs. The stylet was then removed and 5-10 $\mathrm{ml}$ of the contents was aspirated for macroscopic and microscopic examination.

\section{Results}

Clinical findings: Dull demeanor, altered appetite, weight loss and decrease or absence or ruminal motility were recorded in all examined animals. The rectal temperature varied from 38.5 to $40.5^{\circ} \mathrm{C}$. Two cows and 2 buffaloes had recurrent fever. The heart rate was 55 to 100 beats/minute and respiratory rate varied from 15 to 50 breaths/ minute. Conjunctival mucous membrane was congested in two cows and five buffalos, icteric in one cow. Grunting, arched back and other tucked up abdomen were observed in 2 cows. Two cows and two buffaloes had scant hard feces, while one cow and three buffaloes had diarrhea.

Hematological and biochemical findings: The results of complete blood count revealed that all animals had leucocytosis (21.120 \pm 1.630 ; reference range 4.0-12.0 $\left.\mathrm{x} 10^{9} / \mathrm{L}\right)$ and six animals had neutrophilia $(6.50 \pm 1.50$; reference range $\left.0.6-4.0 \times 10^{9} / \mathrm{L}\right)$. Hypoalbuminaemia (14.20 \pm 2.00 ; reference range $21-36 \mathrm{~g} / \mathrm{L})$ and hyperglobulinaemia $(6.36 \pm 0.20$; reference range $36-45 \mathrm{~g} / \mathrm{L})$ were recorded in all animals under investigation. With regard to the liver enzymes, there were mild elevation of ALT ( $60.50 \pm 11.90$; reference range 11-40 units/L) and GGT(24.80 \pm 4.30 ; reference range 6.117.40 units/L) in four animals (3 cattle, one buffalo), while AST, ALP, and CKwere within reference range in all examined animals (AST: $80.64 \pm 20.20$; reference range 78-132 units/L; ALP: $140 \pm 5.50$; reference range 0-500 units/L; CK: $85.40 \pm 10.20$; reference range 35280 units/L). Concerning the renal function, the BUN and creatinine levels were within reference range in all animals (BUN: $7.40 \pm 1.20$; reference range 2.0-7.5 $\mathrm{mmol} / \mathrm{L}$; creatinine: $68.00 \pm 7.00$; reference range 67 $175 \mu \mathrm{mol} / \mathrm{L})$.

Ultrasonographic findings: Hepatic ultrasonographic examination revealed presence of pinpoint echogenic structure in one animal (Figure-1), circumscribed mass with hypoechoic center in 3 animals (Figure-2) and with echogenic or hyperechoic center in 4 (Figure-3) with or without echogenic capsule. The diameters of abscesses varied between pinpoint to $10 \mathrm{~cm}$ in diameter. Centesis of these masses under ultrasonographic guidance yield purulent material wand confirmed as abscesses. Abscesses were located in the right lobe $7^{\text {th }}$ to $12^{\text {th }}$ intercostal spaces, intra-hepatic in 7 animals and peri-hepatic (between liver and reticulum) in one (Figure- 2).

Necropsy and histo-pathological findings ( 3 cases): Liver abscesses are pus-filled, have capsules that vary in thickness, and size . The distribution of abscesses in the liver lobes shows no consistent pattern. Liver contain a single abscess in one case and numerous abscesses in two animals. Abscess was located within hepatic parenchyma in 2 cases (Figure- 4 ) and between liver and reticulum in one case (Figure-5). Histopa- 


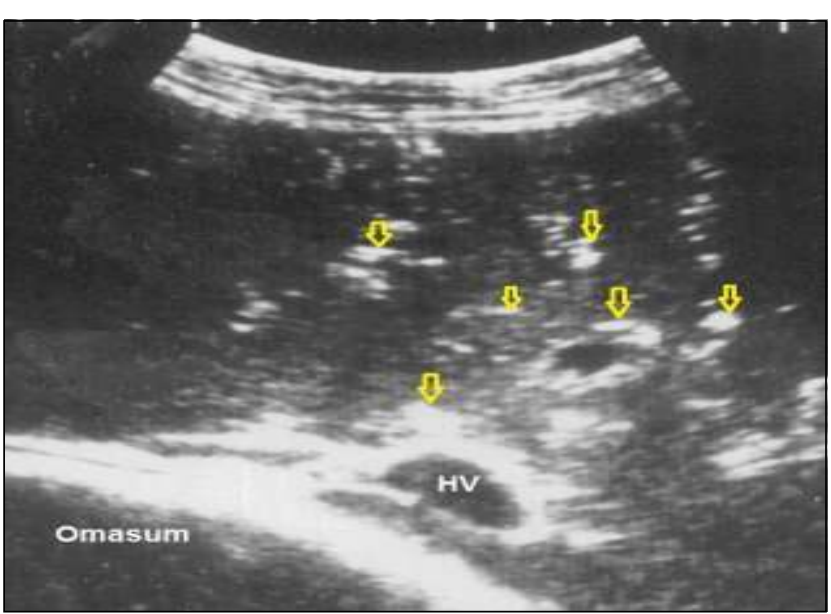

Figure-1: Ultrasonography of liver at right $10^{\text {th }} \mathrm{ICS}$. shows a pinpoint, echogenic structure represent minute abscesses while liver appears as hypoechoic structure.

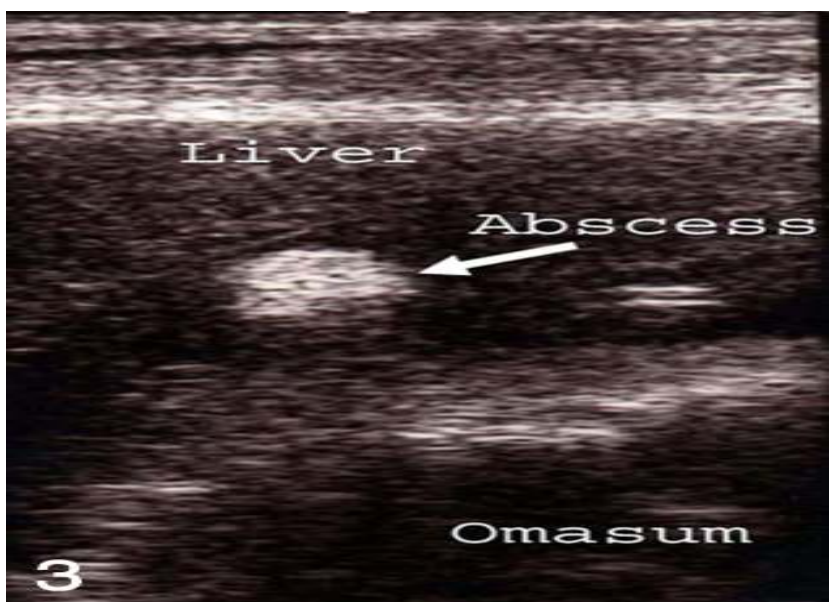

Figure-3: Ultrasonography of liver at right $9^{\text {th }}$ ICS shows a single abscess as circumscribed hyperechoic mass while liver appears as hypoechoic structure.

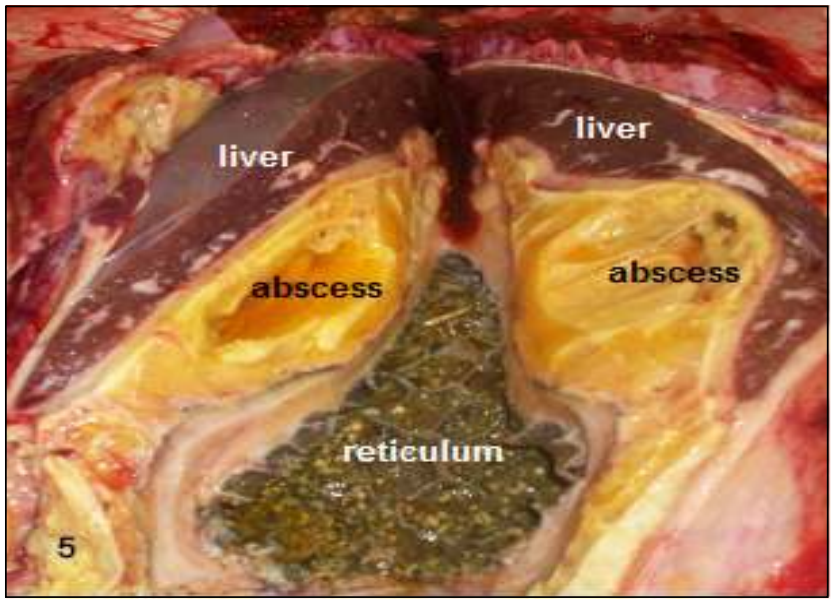

Figure-5: Post mortem finding of a buffalo shows a cut section in abscess with yellow capsule between liver and reticulum (cut section)

thologically, liver abscesses appeared with a necrotic center containing leukocytes, hepatocytes and cellular debris (Figure- 6).

\section{Discussion}

Liver is highly susceptible for parenchymal, vascular and biliary system lesions. Bacterial, chemical, viral,

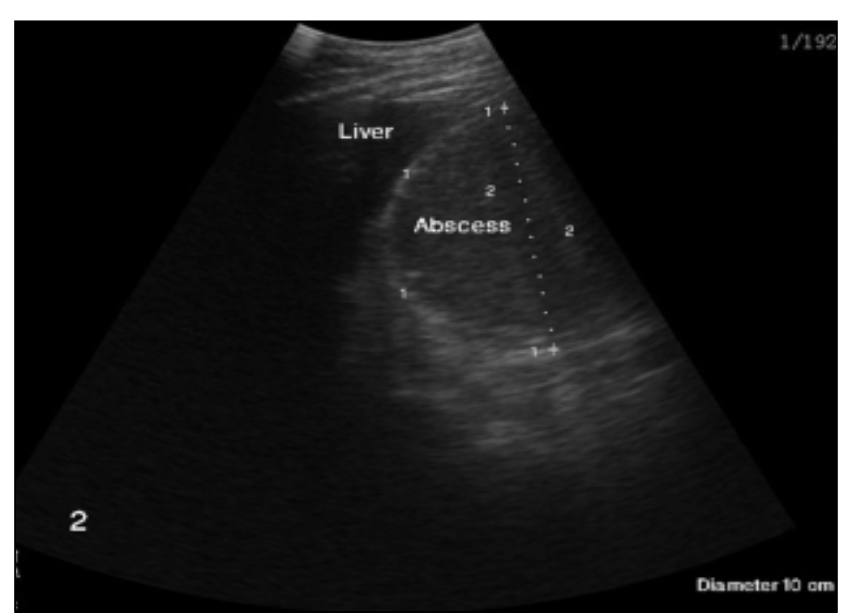

Figure-2: Ultrasonography of liver at right $7^{\text {th }}$ ICS shows a large single abscess with $10 \mathrm{~cm}$ diameter appears as circumscribed hypoechoic mass (2) surrounded by echogenic capsule (1) locates between liver and reticulum.

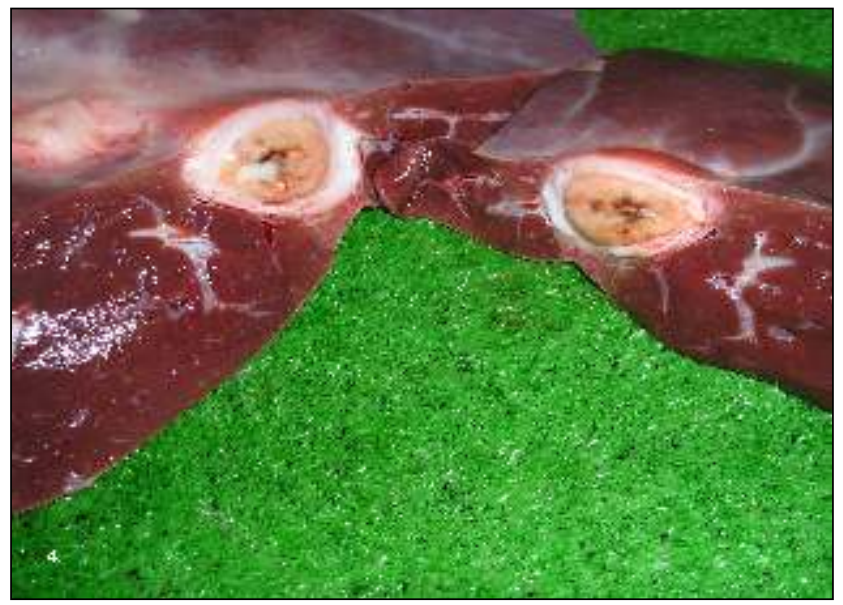

Figure-4: Post mortem finding of a cattle shows a cut section in abscess which appears as circumscribed pus-filled structure with a thick capsule.

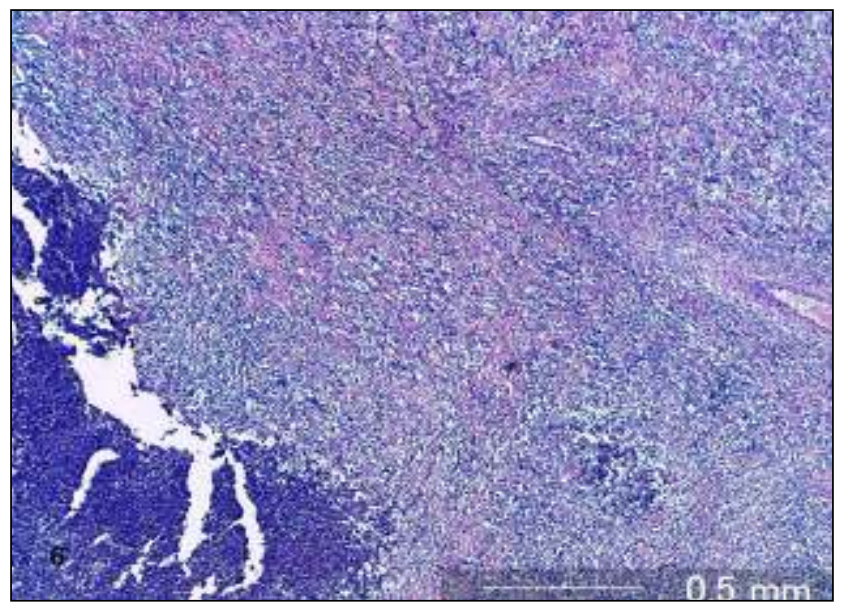

Figure-6: Histopathology of an abscess showing a necrotic center containing leukocytes, hepatocytes and cellular debris. $H \& E \times 40$.

toxic or immune-mediated insults may cause focal or diffuse hepatic abnormalities or lesions [19].

Clinical findings: All diseased animals appeared with dull demeanor, altered appetite, weight loss and decrease or absence or ruminal motility. Recurrent fever, abdominal pain and congestion and or icteric mucous membrane 
were also observed but with variable degrees. These signs are not specific signs and considered as general signs for many diseases. These results are partially similar to those described previously $[5,6]$ in cattle. Other report [19] stated that cattle with liver abscesses seldom exhibit any clinical signs and abscesses can be detected only at the time of slaughter.

Hematological and biochemical findings: The hematological picture of diseased animals revealed leucocytosis in all animals with neutrophilia in six. These results are in consistent with those obtained previously [20] in Holstein dairy cattle. Moreover, Hypoalbuminaemia with hyperglobulinaemia were reported in all diseased animals under investigation. These results are in accordance with those obtained previously in cattle $[18,21]$ and in camel [22].

With regard to the liver enzymes (AST, ALT, ALP, $\mathrm{CK}$ and GGT) and renal function parameters, BUN and creatinine were within normal range in our study, only mild elevation in ALT and GGT were detected in 4 animals. These results are in agreement with El-Sabban et al [7]. Generally, hematology and liver function tests are not reliable indicators of liver abscesses [7, 23].

Ultrasonographic findings: In contrary to the hematological and biochemical findings, the ultrasonographic result were of considerable value for diagnosing liver abscesses although, the ultrasonographic scanning cannot visualize the whole liver. The usefulness of the technique for diagnosis of various liver abnormalities including abscesses in animals has been well documented $[6,8]$.

In the present study, liver abscesses varied from hypoechoic to hyperechogenic with or without a capsule. The presence of a capsule, considerable size of the abscess and the variation in echogenicity provide an idea about the duration of abscess development. In the early stage of abscess development, ultrasonography revealed that an abscess had no capsule with anechoic content, as the disease progress abscess become mature and appeared as hypoechoic to hyperechogenic mass $[6,18]$. In one buffalo, abscess with $10 \mathrm{~cm}$ diameter was detected between liver and reticulum. This result could attribute to formation of abscess as sequelae of reticular foreign body. Similar results have been reported previously $[24,25]$.

Necropsy and histo-pathological findings: In the present study, liver abscesses appeared as pus-filled, have capsules that vary in thickness, and size. The distribution of abscesses in the liver lobes shows no consistent pattern. Histologically, abscesses appeared with a necrotic center containing leukocytes, hepatocytes and cellular debris. During the process of liver abscess development, the earliest lesion is a micro abscess, possibly induced by an embolus of bacteria in the hepatic sinusoid; the lesion then progresses to coagulative necrosis by involving adjacent hepatocytes and subsequently, the lesion gradually changes into a pus-filled, encapsulated, true abscess [26, 27].

\section{Conclusion}

Ultrasonography combined with fine-needle aspiration yielded diagnosis and helped in determining the duration, size and location of hepatic abscess which could not be detected by clinical or hemato-biochemical findings.

\section{Authors' contributions}

AMA and SMG made available relevant literatures and conducted examinations. MT participated in sampling and supervised the research. All authors participated in draft and revision of the manuscript. All authors read and approved the final manuscript.

\section{Acknowledgments}

Special thanks to Animal Medicine Department, Faculty of Veterinary Medicine, Zagazig University, Zagazig, Egypt that supported us during conduction of this research. This study was funded by authors and no outside fund was received.

\section{Competing interests}

The authors declare that they have no competing interests.

\section{References}

1. Radostits, O.M., Gay, C.C., Blood, D.C. and Hinchcliff, K.W. (2007) A Textbook of the Diseases of Cattle, Sheep, Pigs, Goats and Horses. W.B. Saunders, London. p393-395.

2. Scott, $\mathrm{P}(2013)$ Diagnosis and treatment of liver abscesses in cattle. Livest. Sci., 18(2): 20-23.

3. Nagaraja, T.G. and Lechtenberg, K.F. (2007) Liver Abscesses in Feedlot Cattle: Review article. Vet Clin North Am Food Anim Pract, 23: 351-369.

4. Zinn, R. A., and Plascencia, A. (1996) Effects of forage level on the comparative feeding value of supplemental fat in growing finishing diets for feedlot cattle. J. Anim. Sci. 74:1194-1201.

5. Andrews, A.H., Blowey, R.W., Boyd, H. and Eddy, R.G. (2004) Bovine Medicine, Diseases and Husbandry of Cattle. 2nded. Ames, Iowa: Blackwell Science Ltd, p835-839.

6. Braun, U., Pusterla, N. and Wild, K. (1995) Ultrasonographic findings in 11 cows with a hepatic abscess. Vet Rec 137: 284-290.

7. El-Sabban, F.F., Rothenbacher, H., Long, T.A. and Baumgardt, B.R. (1971) certain blood constituents and serum transaminases in Hereford steers fed high-energy rations. Am J Vet Res 32: 1027-1032.

8. Lechtenberg, K. F., and Nagaraja, T. G. (1991) Hepatic ultrasonography and blood changes in steers with experimentally induced liver abscesses. Am. J. Vet. Res. 52: 803-809.

9. Streeter, R.N. and Step, D.L. (2007) Diagnostic ultrasonography in ruminants. Vet Clin North Am Food Anim Pract, 23: 541-574.

10. Braun, U. (2009) Ultrasonography of the Liver in Cattle. Vet Clin North Am Food Anim Pract, 25: 591-609.

11. Komeilian, M.M., Sakha, M., Nadalian, M.G. and Veshkini, A. (2011) Hepatic Ultrasonography of Dairy Cattle in Postpartum Period : Finding the Sonographic Features of Fatty Liver Syndrome. Aust J Basic Appl Sci., 5: 701-706.

12. McKaigeny, C. (2013) Hepatic Abscess: Case repot and review. West J Emerg Med. 14(2): 154-157.

13. Bakoyiannis, A., Delis, S., Triantopoulou, C. and Dervenis, C. (2013) Rare cystic liver lesions: A diagnostic and managing challenge. World J Gastroenterol; 19(43): 76037619.

14. Kumar, V., Kumar, A., Varshney, A. C., Tyagi, S. P, Kanwar, 
M. S. and Sharma, S. K. (2012) Diagnostic Imaging of Canine Hepatobiliary Affections: A Review," Vet. Med. Int., vol. 2012, Article ID 672107, 15 pages, 2012. doi:10.1155/ 2012/672107.

15. Hussein, H.A. and Elrashidy, M. (2014) Ultrasonographic features of the liver with cystic echinococcosis in she. Vet Rec Open 2014;1:e00004.

16. Robinson, E. and Sprayberry, K.M. (2009) Current therapy in equine medicine. $6^{\text {th }}$ edn. Saunders El Sevier. p170.

17. Rosenberger, G. (1990) Die Klinische Untersuchung des Rindes. $3^{\text {rd }}$ edn. Berlin and Munich, Paul Parey. p10-30.

18. Mohamed, T. and Oikawa, S. (2007) Ultrasonographic characteristics of abdominal and thoracic abscesses in cattle and buffaloes. J Vet MedA 54: 512517.

19. Nagaraja, T.G., Laudert, S.B. and Parrott, J.C. (1996) Liver abscesses in feedlot cattle. Part 2. Incidence, economic importance and prevention. Comp Cont Edu. Pract. Vet 18: S264-S273.

20. Doré, E., Fecteau, G., Hélie, P. and Francoz, D. (2007) Liver abscesses in Holstein dairy cattle: 18 cases (1992-2003). $J$ Vet. Intern. Med., 21(4):853-6.

21. Craig, A.M., Pearson, E.G. and Rowe, K. (1992) Serum bile acid concentrations in clinically normal cattle: comparison by type, age, and stage of lactation. Am J Vet Res 53: 1784-
1786.

22. El-Deeb, W.M. and Fouda, T.A. (2013) Liver Abscesses in Dromedary Camels (Camelus dromedaries): Oxidative Stress Biomarkers and Proinflammatory Cytokines. $J$ Vet. Sci Technol., 4: 140.

23. Kaneko, J. J., Harvey, J. W. and Bruss, M. L. (1997) Clinical Biochemistry of Domestic Animals, 5th edn, New York, Academic Press, p885-905.

24. Braun U, Iselin U, Lischer C, Fluri E (1998): Ultrasonographic findings in five cows before and after treatment of reticular abscesses. Vet Rec., 142:184-189.

25. Abdelaal, A. M., Floeck, M., Maghawry, S. E. and Baumgartner, W. (2009) Clinical and ultrasonographic differences between cattle and buffaloes with various sequelae of traumatic reticuloperitonitis. Veterinaria Medicina 54: 399-406.

26. Lechtenberg, K. F., Nagaraja, T. G., Leipold, H. W. and Chengappa, M. M. (1988) Bacteriologic and histologic studies of hepatic abscesses in cattle. Am. J. Vet. Res. 49:5862 .

27. Tehrani, A., Javanbakht, J., Hassan, M., Zamani, M., Rajabian, M., Akbari, H. and Shafe, R. (2012) Histopathological and Bacteriological Study on Hepatic Abscesses of Herrik Sheep. J Med Microb Diagn. 1: 115.

$* * * * * * * *$ 